\title{
Metastatic adenocarcinoma to the breast from the lung simulates primary breast carcinoma-a clinicopathologic study
}

\author{
Xin Wang ${ }^{1 \#}$, Yang Luo ${ }^{2 \#}$, Li Liu ${ }^{1}$, Jiacong Wei ${ }^{1}$, Huizi Lei ${ }^{1}$, Susheng Shi ${ }^{1}$, Lin Yang ${ }^{1}$ \\ ${ }^{1}$ Department of Pathology, National Cancer Center/National Clinical Research Center for Cancer/Cancer Hospital, Chinese Academy of Medical \\ Sciences and Peking Union Medical College, Beijing, China; ${ }^{2}$ Department of Medical Oncology, National Cancer Center/National Clinical Research \\ Center for Cancer/Cancer Hospital, Chinese Academy of Medical Sciences and Peking Union Medical College, Beijing, China \\ Contributions: (I) Conception and design: L Yang, X Wang, Y Luo; (II) Administrative support: L Yang, Y Luo; (III) Provision of study materials or \\ patients: L Yang, X Wang, Y Luo; (IV) Collection and assembly of data: L Yang, X Wang; (V) Data analysis and interpretation: L Yang, X Wang; (VI) \\ Manuscript writing: All authors; (VII) Final approval of manuscript: All authors. \\ "These authors contributed equally to this work. \\ Correspondence to: Lin Yang. Department of Pathology, National Cancer Center/Cancer Hospital, Chinese Academy of Medical Sciences and Peking \\ Union Medical College, No. 17 Panjiayuan Nanli, Chaoyang District, Beijing 100021, China. Email: linyang0616@126.com.
}

Background: Rare extra-mammary metastases of adenocarcinoma to the breast closely mimic primary invasive breast carcinoma (PBC), and specifically without an aware of clinical history, pose a difficult diagnostic issue.

Methods: With the aim to improve differential diagnosis of lung adenocarcinoma metastasis and primary breast carcinoma in the breast, we retrieved 41 breast metastases from lung adenocarcinoma, seven of which were from the archived pathologic files of Cancer Hospital, Chinese Academy of Medical Science (CHCAMS) between 2001 and 2019, and the other 34 cases were collected from the published literatures. Clinicopathological features were collected and analyzed for differential diagnosis of primary lung malignancy, triple negative breast pathology and breast lesions without ipsilateral axillary lymphadenopathy or with contralateral axillary lymphadenopathy. Supplementary breast (GCDFP-15, or GATA-3) and lunglineage (TTF-1) immunostaining plus genetic alternation analysis were also recorded and analyzed.

Results: Among the 41 cases, there were 37 females and four males, with a median age of 63 (range, 4081 ) years at diagnosis of the breast lesion. Twenty-four cases $(58.5 \%, 24 / 41)$ were detected metachronously to the counterpart of the lung. Strikingly, 13 cases $(31.7 \%, 13 / 41)$ were initially misdiagnosed as primary breast cancer, and differential diagnostic factors were compared and analyzed between the correct and misdiagnosed cases, among which a documentation of lung cancer history showed significant difference. Pathologist initially misinterpreted six cases $(46.2 \%, 6 / 13)$ as PBC on needle biopsy of breast mass with an unknown lung cancer history. The clinical diagnosis was considered two cases $(15.4 \%, 2 / 13)$ to be either a primary breast tumor with lung and pleural metastasis or two synchronous primary tumors. Three cases $(23.1 \%, 3 / 13)$ were initially misinterpreted as PBC by breast ultrasonography. TTF-1 immunostaining was found to be critical for a correct diagnosis of metastatic lesion $(84.6 \%, 11 / 13)$ from the initially misdiagnosed cases as PBC.

Conclusions: Metastatic lung adenocarcinoma to the breast, although rare, should be considered in the differential diagnosis of primary breast carcinoma, especially when the breast lesion exhibits as a "triplenegative invasive carcinoma". A documented lung cancer history combined with the clinicoradiological assessment and pathological evaluation are essential to make a correct differential diagnosis. TTF-1 immunostaining is crucial in approaching the diagnosis.

Keywords: Lung adenocarcinoma; breast metastases; breast carcinoma/secondary; differential diagnosis

Submitted Jun 03, 2020. Accepted for publication Jan 22, 2021.

doi: $10.21037 /$ tcr-20-2250

View this article at: http://dx.doi.org/10.21037/tcr-20-2250 


\section{Introduction}

Primary advanced adenocarcinoma of the lung is prone to distant metastasis, with the most common metastatic organs being lung, liver, adrenal gland, brain, bone etc. (1). According to sporadic case reports, the breast was reported as a metastatic site for primary lung adenocarcinomas and the incidence of breast metastases from an extramammary primary tumor can be as low as $0.5-3.0 \%(2-7)$. Because of its rarity, even with known history of lung cancer, breast metastases would have been easily misdiagnosed as primary breast cancer and thus leads to unnecessary mastectomies $(6,8,9)$. Due to the distinct biological mechanisms, advanced lung adenocarcinoma and breast cancer are treated with different therapies, such as EGFR tyrosine kinase inhibitors (TKIs) for lung cancer, and estrogen receptor (ER), progesterone receptor $(\mathrm{PR})$ related endocrine treatment or human epidermal growth factor receptor 2 (HER-2) related target therapy for breast cancer. Therefore, for patients with lung cancer history who have synchronous or heterochronous breast nodules, it is necessary to fully identify whether it is a metastatic lesion or a secondary primary breast tumor.

The main reason for misdiagnosis lies in the fact that breast metastasis of lung adenocarcinoma shares similar cellular morphology as primary invasive ductal carcinoma of breast, especially in samples from fine needle biopsies.

In this study, we aim to improve the differential diagnosis criteria of primary breast nodules and breast nodules metastasized from lung adenocarcinoma by retrieving and analyzing 41 cases of metastatic adenocarcinoma from the lung to breast. We made a comparative analysis from the perspective of the accuracy of the first diagnosis in order to explore which factors affect the accuracy of pathological diagnosis. A documented lung cancer history combined with the clinicoradiological assessment and pathological evaluation are essential to make a correct differential diagnosis. We present the following article in accordance with the STROBE reporting checklist (available at http:// dx.doi.org/10.21037/tcr-20-2250).

\section{Methods}

Metastatic cases of lung adenocarcinoma to the breast were retrieved both from Department of Pathology in Cancer Hospital, Chinese Academy of Medical Science (CHCAMS) and published literatures from 2001 to 2019. Computerized literature searches were performed using PubMed databases. $\mathrm{MeSH}$ terms and free words were all used. The search terms were as follows: "breast cancer", "lung cancer", "lung carcinoma", "adenocarcinoma", "metastasis", "lung cancer/ primary", "breast cancer/secondary", "metastasis to the breast" and "extramammary primary". We also screened the reference lists of all relevant articles for potentially suitable studies. In this way, we collected seven archived cases from CHCAMS and 34 counterparts from literatures, respectively. The clinicopathological related features were collected and recorded, including gender, age at diagnosis of the breast lesions, possible prior history of a lung primary before the breast disease, solitary or multicentric masses, lymphadenopathy region, concomitant metastases to other organs than breasts, etc. Specially, the archived pathology reports and slides of the selected cases from CHCAMS were reviewed by two pathologists (XW and LY) to confirm the diagnosis and clarify the pathological features for differential diagnosis. Immunohistochemical markers were also collected and analyzed in which lineage-specific stains for lung carcinoma [thyroid transcription factor-1, TTF1 (SP141, Roche Diagnostics, China)] and those for the breast [gross cystic disease fluid protein, GCDFP-15 (23A3, Maixin, China) or transcription factor, GATA-3 (EP368, Zhongshan, China)] as well as ER (SP1, Roche Diagnostics, China), PR (1E2, Roche Diagnostics, China) and HER-2 (4B5, Roche Diagnostics, China). Genetic alternation status (EGFR mutation, $A L K$ gene rearrangement, etc.), if available, was also collected. The study was conducted in accordance with the Declaration of Helsinki (as revised in 2013). The study was approved by the Ethics Committee board of National Cancer Center/National Clinical Research Center for Cancer/Cancer Hospital (NCC-004409) and individual consent for this retrospective analysis was waived.

\section{Statistical analysis}

The Chi-square test was performed to clarify the various significances of the above factors among initial misdiagnosis cases or no misdiagnosis cases. $\mathrm{P}<0.05$ was considered as a statistically significant result.

\section{Results}

\section{Clinicopathological features of lung adenocarcinomas to the breast, retrieved from CHCAMS and literatures}

In totally, we collected 41 cases of metastatic lung adenocarcinomas to the breast, seven of which were from the archived files of the Department of Pathology in CHCAMS; the other 34 cases were from 21 published 
case reports or mini-review between 1992 and 2019. The clinicopathological features for all 41 cases were listed in Table 1. Among the 41 cases, there are 37 females and 4 males, with a median age of 63 (range, 40-81) years at diagnosis of the breast lesion. Twenty-four cases $(58.5 \%$, 24/41) were metachronous to the primary lung tumors, while $17(41.5 \%, 17 / 41)$ were detected synchronously. Thirty-one $(75.6 \%, 31 / 41)$ cases were detected as a solitary metastatic nodule, and ten $(24.4 \%, 10 / 41)$ were detected multifocal nodules including three with bilateral breast involvement. Tumor border on radiographical examination was recorded in Table 1, among which 31 cases $(75.6 \%$, $31 / 41)$ showed an irregular border, while ten cases $(24.4 \%$, $10 / 41$ ) showed a regular border. Twenty of the 41 cases were documented with a previous treatment for primary lung cancer, 17 cases of which received systemic chemotherapy and/or radiation, compared with three instances of segmental mastectomy. Forty $(97.6 \%, 40 / 41)$ patients had lymph node involvement at the time of diagnosis, in which 28 patients had axillary lymph node metastasis, eight with bilateral axillary lymph nodes involvement, and 24 with hilar/mediastinal lymph node involvement. Twentyfour $(58.5 \%, 24 / 41)$ patients showed distant metastases to other organs at the time of the breast mass detection. The metastasized organs were bone $(\mathrm{n}=13)$, liver $(\mathrm{n}=6)$, pleura $(n=5)$, brain $(n=5)$, adrenal gland $(n=4)$, subcutaneous $(n=4)$, pancreas $(n=2)$, and choroid plexus $(n=1)$.

\section{Clinicopathological features of initial diagnosis retrieved from CHCAMS and literatures}

Thirteen cases $(31.7 \%, 13 / 41)$ were initially misdiagnosed as primary breast cancer. The clinical diagnosis was considered cases of No. 37 and 39 (15.4\%, 2/13) in Table 1

Table 1 Clinicopathological features of lung adenocarcinomas to the breast, retrieved from CHCAMS and literatures ( $\mathrm{n}=41)$

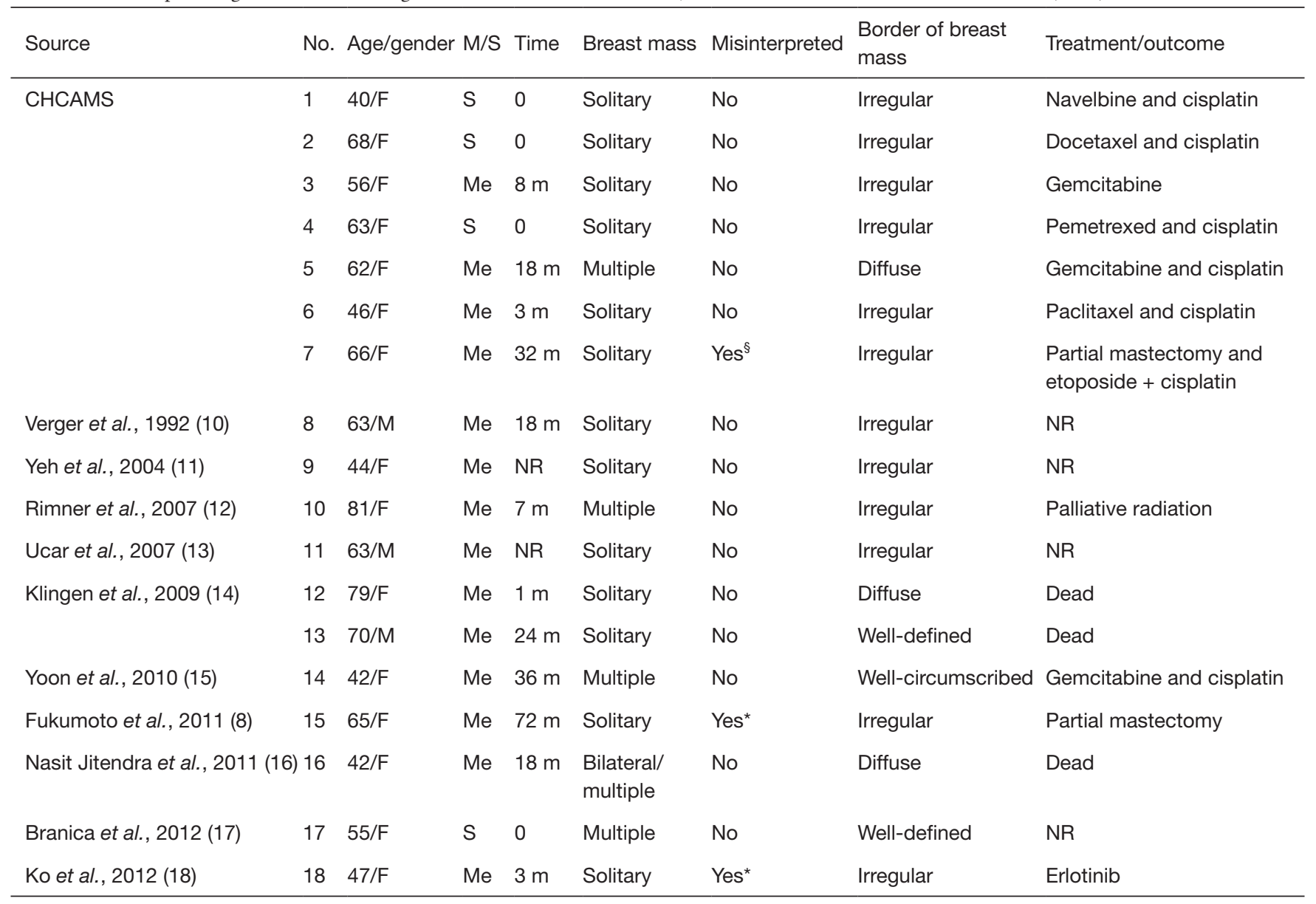

Table 1 (continued) 
Table 1 (continued)

\begin{tabular}{|c|c|c|c|c|c|c|c|c|}
\hline Source & No. & Age/gender & $\mathrm{M} / \mathrm{S}$ & Time & Breast mass & Misinterpreted & $\begin{array}{l}\text { Border of breast } \\
\text { mass }\end{array}$ & Treatment/outcome \\
\hline Sato et al., 2012 (19) & 19 & $57 / F$ & $\mathrm{Me}$ & $12 \mathrm{~m}$ & Solitary & No & Irregular & $\begin{array}{l}\text { CBDCA + pemetrexed + } \\
\text { bevacizumab }\end{array}$ \\
\hline Mirrielees et al., 2014 (20) & 20 & $58 / F$ & $\mathrm{Me}$ & $36 \mathrm{~m}$ & Solitary & Yes $^{\#}$ & Irregular & Erlotinib \\
\hline \multirow[t]{8}{*}{ Ali et al., 2018 (9) } & 21 & $64 / F$ & $\mathrm{Me}$ & $36 \mathrm{~m}$ & Solitary & $Y_{e s}^{\S}$ & Circumscribed & NR \\
\hline & 23 & $45 / F$ & $\mathrm{Me}$ & NR & Solitary & No & Irregular & NR \\
\hline & 24 & $70 / F$ & $\mathrm{Me}$ & NR & Solitary & Yes $^{*}$ & Circumscribed & Partial mastectomy \\
\hline & 25 & $69 / F$ & $\mathrm{Me}$ & NR & Solitary & Yes $^{\&}$ & Infiltrative & NR \\
\hline & 26 & $64 / F$ & $\mathrm{Me}$ & NR & Solitary & No & Irregular & NR \\
\hline & 29 & $63 / F$ & $\mathrm{~S}$ & 0 & Bilateral/two & $Y_{e s}^{\S}$ & Infiltrative & NR \\
\hline & 30 & $65 / F$ & $S$ & 0 & Solitary & No & Circumscribed & NR \\
\hline & 31 & $65 / F$ & $\mathrm{~s}$ & 0 & Solitary & $Y_{e s}^{\S}$ & Irregular & NR \\
\hline Sadikot et al., 1997 (21) & 32 & $47 / F$ & $\mathrm{~S}$ & 0 & Multiple & No & Well-circumscribed & Dead \\
\hline Babu et al., 2009 (6) & 33 & $51 / F$ & $\mathrm{~S}$ & 0 & Solitary & $Y_{e s}^{\S}$ & Irregular & $\begin{array}{l}\text { Wide local excision and } \\
\text { axillary node clearance }\end{array}$ \\
\hline Maounis et al., 2010 (22) & 34 & $73 / F$ & S & 0 & Solitary & No & Irregular & $\begin{array}{l}\text { Bevacizumab, cisplatin } \\
\text { and docetaxel }\end{array}$ \\
\hline \multirow[t]{2}{*}{ Wu et al., 2019 (26) } & 39 & $49 / F$ & $S$ & 0 & Multiple & Yes $^{\star \star}$ & Well-defined & Crizotinib \\
\hline & 40 & $63 / F$ & $\mathrm{Me}$ & $15 \mathrm{~m}$ & $\begin{array}{l}\text { Bilateral/ } \\
\text { multiple }\end{array}$ & No & Irregular & Crizotinib \\
\hline Erhamamci et al., 2016 (27) & 41 & $74 / \mathrm{M}$ & $S$ & 0 & Solitary & No & Irregular & Dead \\
\hline
\end{tabular}

\footnotetext{
$\S$, Pathologist initially misinterpreted as PBC on needle biopsy of breast mass with an unknown lung cancer history, until the patient came back with a supplementation of a 32 months' lung cancer history. Later on, TTF-1 immunostaining was applied and the diagnosis was corrected to be a metastatic one. *, Initially misinterpreted as PBC by breast ultrasonography. Case 24 had a solitary circumscribed 2-cm mass in the right breast that appeared benign on ultrasound and mammography with the differential diagnosis of a cyst vs. fibroadenoma. **, The clinical diagnosis was considered to be either a primary breast tumor with lung and pleural metastasis or two synchronous primary tumors. ${ }^{\&}$, This patient had a left breast mass with contralateral enlarged axillary lymph nodes and mediastinal lymphadenopathy. Nine years earlier, she had a history of right mastectomy for ductal carcinoma. This case was referred to the cancer agency and TTF-1 was later found to be positive. ", This patient's family history was significant for a sister diagnosed with breast cancer in her forties and her mother's diagnosis of breast cancer in her sixties. Later, a repeat pathologic evaluation of the slides from the patient's breast biopsy was requested. Immunolabeling for TTF-1 demonstrated the tissue to be more consistent with a metastatic lung adenocarcinoma. M, male; F, female; Me, metachronous; S, synchronous; Time, interval time; Misinterpreted, initially misinterpreted as PBC; NR, not retrievable; PBC, primary invasive breast carcinoma.
} 
Table 2 Clinicopathological features of initial diagnosis retrieved from CHCAMS and literatures $(\mathrm{n}=41)$

\begin{tabular}{|c|c|c|c|c|}
\hline Features & Misdiagnosis* $(n=13), n(\%)$ & No misdiagnosis $(n=28), n(\%)$ & $\chi^{2}$ & $P$ \\
\hline CHCAMS & $1(7.7)$ & $6(21.4)$ & & \\
\hline Literature & $12(92.3)$ & $22(78.6)$ & & \\
\hline Known tumor history & & & 15.138 & $<0.001$ \\
\hline No & $6(46.2)$ & $0(0.0)$ & & \\
\hline Time interval & & & 0.173 & 0.678 \\
\hline Synchronous & $6(46.2)$ & $11(39.3)$ & & \\
\hline Metachronous & $7(53.8)$ & $17(60.7)$ & & \\
\hline Multiple & $3(23.1)$ & $7(25.0)$ & & \\
\hline Specimen type & & & 1.086 & 0.297 \\
\hline Biopsy & $10(76.9)$ & $25(89.3)$ & & \\
\hline Mastectomy & $3(23.1)$ & $3(10.7)$ & & \\
\hline Ipsilateral axillary $\#$ & & & 2.342 & 0.126 \\
\hline Yes & $11(84.6)$ & $17(60.7)$ & & \\
\hline No & $2(15.4)$ & $11(39.3)$ & & \\
\hline Distant ${ }^{*}$ & & & 0.777 & 0.378 \\
\hline
\end{tabular}

*, Initially misdiagnosed as primary breast carcinoma. ${ }^{\#}$, Ipsilateral axillary lymphadenopathy to the breast mass. ${ }^{*}$, Distant lymphadenopathy (including contralateral axillary).

to be either a primary breast tumor with lung and pleural metastasis or two synchronous primary tumors. In Table 1 cases of No. 15, 18 and $24(23.1 \%, 3 / 13)$ were initially misinterpreted as primary invasive breast carcinoma (PBC) by breast ultrasonography. Cases of No. 25 in Table 1 had a left breast mass with contralateral enlarged axillary lymph nodes and mediastinal lymphadenopathy. Nine years earlier, she had a history of right mastectomy for ductal carcinoma. This case was referred to the cancer agency and TTF- 1 was later found to be positive. The other patient's family history which was cases of No. 20 in Table 1 was significant for a sister diagnosed with breast cancer in her forties and her mother's diagnosis of breast cancer in her sixties. Later, a repeat pathologic evaluation of the slides from the patient's breast biopsy was requested. Immunolabeling for TTF-1 demonstrated the tissue to be more consistent with a metastatic lung adenocarcinoma. Pathologist initially misinterpreted cases of No. 7, 21, 27, 29, 31 and $39(46.2 \%, 6 / 13)$ in Table 1 as PBC on needle biopsy of breast mass with an unknown lung cancer history, until the patient came back with a supplementation of lung cancer history. Later on, TTF-1 immunostaining was applied and the diagnosis was corrected to be a metastatic one. A comparative analysis was made in Table 2 to clarify the associated clinicopathological features with respect to misdiagnosis. A history of lung cancer showed significant difference between initial correct diagnosis and misdiagnosis (28/28, 100\% vs. 7/13, 53.8\%; $\mathrm{P}<0.001)$ (Table 2).

\section{Immunobistochemistry (IHC) of initial diagnosis retrieved from CHCAMS and literatures}

Available IHC staining for differential diagnosis were 
Table 3 IHC of initial diagnosis retrieved from CHCAMS and literatures $(\mathrm{n}=41)$

\begin{tabular}{lcc}
\hline IHC markers & $\begin{array}{r}\text { Misdiagnosis* } \\
(\mathrm{n}=13), \mathrm{n}(\%)\end{array}$ & $\begin{array}{c}\text { No misdiagnosis } \\
(\mathrm{n}=28), \mathrm{n}(\%)\end{array}$ \\
\hline ER $(+)$ & 0 & 0 \\
PR $(+)$ & 0 & 0 \\
HER-2 (+) & 0 & 0 \\
Mammaglobin, & 0 & 0 \\
GCDFP-15, GATA-3 (+) & $11(84.6)^{\#}$ & 27 (96.4) \\
TTF-1 (+)
\end{tabular}

*, Initially misdiagnosed as PBC. ", TTF-1 was found to be positive after the 11 cases of 13 cases were initially misdiagnosed as PBC. The other two cases of 13 cases initially misdiagnosed as PBC (cases of No. 21 and 31) were found to be negative for TTF-1 which compounded the problem. In view of the known lung cancer history that preceded the development of a breast mass, widespread disease, and negative breast markers and hormone receptors, the breast tumor was considered a TTF-1 negative metastatic adenocarcinoma of lung. ER, estrogen receptor; PR, progesterone receptor; HER2, human epidermal growth factor receptor 2; TTF-1, thyroid transcription factor-1; GCDFP-15, gross cystic disease fluid protein; GATA-3, transcription factor; PBC, primary invasive breast carcinoma.

shown in Table 3. No positive staining of ER, PR, HER2, mammaglobin, GCDFP-15, or GATA-3 was found in all confirmed metastatic cases. The positive rate of TTF1 in initially correct diagnosis cases was $96.4 \%$ (27/28). Besides, TTF-1 was found later to be positive in 11 cases of the 13 initially misdiagnosed as PBC $(11 / 13,84.6 \%)$ and thus helped correcting the initial misdiagnosis. Cases of No. 21, 26 and 31 were considered a TTF1-negative metastatic adenocarcinoma of the lung in view of the known lung cancer history that preceded the development of a breast mass, widespread disease, and negative breast markers and hormone receptors. There were nine cases with reported genetic detections, including two p.L858R in $E G F R$ exon 21, four EGFR exon 19 deletion, two with $A L K$ rearrangement, and one with ROS1 rearrangement.

\section{Discussion}

In female patients, lung adenocarcinoma and breast carcinoma are two most common types of malignancies. They share similar organs of distant metastasis, including bone, liver and the brain. Unlike the lung, the breast rarely raises concern as a metastatic site. Rarity of breast involvement by metastatic tumors is reported due to large areas of fibrous tissue, relatively poor blood supply and anatomically non-connected locations (5).

Unlike lung squamous cell carcinoma or small cell lung cancer, pulmonary adenocarcinoma metastasis to the breast is more difficult to be distinguished from primary breast cancer due to the similar morphological features they shared. Moreover, the inadequate sample size from the commonly applied fine needle biopsy may lead to lack of specific histological images such as comedo necrosis in breast carcinoma. Thus, there is a big challenge in differential diagnosis.

Since the imaging manifestations or clinical symptoms of the metastatic lesion are heterogenous between patients, it is hard to make a correct diagnosis from these aspects other than a pathological one $(28,29)$. This was exemplified by cases of No. 15, 18 and 24, which were initially misinterpreted as PBC by breast ultrasonography. Furthermore, case No. 24 had a solitary circumscribed mass with a diameter of $2.0 \mathrm{~cm}$ in the right breast that appeared benign on ultrasound and mammography, favoring of a clinical diagnosis of a cyst or fibro adenoma. Besides, case No. 37 and 39 were initially misinterpreted to be primary breast tumor from the clinical perspective. Therefore, the final of differential diagnosis should be decided by the pathologists with additional documentation on lung cancer history provided by responsible physicians.

Histologically, some indicators may help to identify the metastatic adenocarcinomas and PBCs. First, elastosis is a consistent indicator of primary neoplasm but is rarely observed in secondary tumors (3). Other studies described a sharp transition at the border of the lesion and the tumor presence in the subcutaneous, rather than parenchymal breast tissue $(30,31)$. Also noted in our series, metastatic tumors to the breast frequently showed irregular tumor borders and commonly present as a solitary (rather than multicentric) breast mass. The absence of in situ carcinoma strongly supports a metastatic tumor, although it may not occur in all primary invasive carcinomas $(22,32)$. Most researchers agree that calcifications and microcalcifications except for ovarian serous carcinoma are extremely rare and observed only in the patients with metastatic papillary carcinoma with psammoma bodies (32-34). However, for samples from fine needle aspiration, a correct differential diagnosis is more challenging, especially for those patients without a documented history of lung cancer (33-35). The authors experienced one such case (No. 7 in Table 1), which was a needle biopsy 


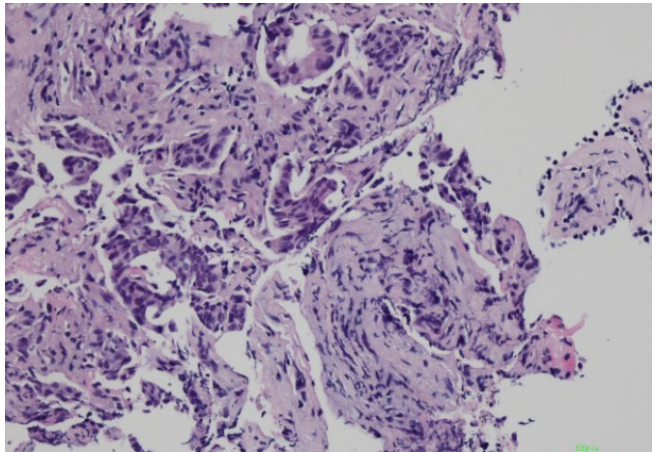

Figure 1 Lung biopsy of case 7 (H-E, ×20). Primary lung adenocarcinoma is indicated by red arrow, as compared with the normal alveolar epithelium (seen in black arrow).

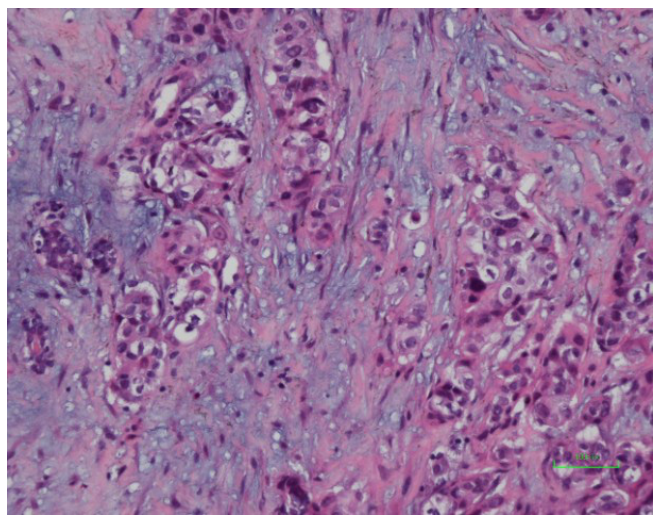

Figure 2 Breast biopsy of case 7. (H-E, $\times 20)$. The red arrow indicates adenocarcinoma. The black arrow indicates normal mammary duct.

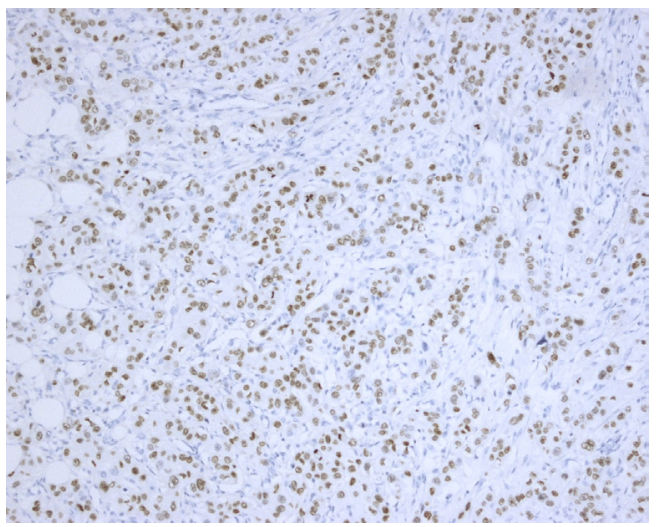

Figure 3 Breast segmental resection of case 7. Nuclear positivity for TTF-1 (IHC, ×20). IHC, immunohistochemistry.

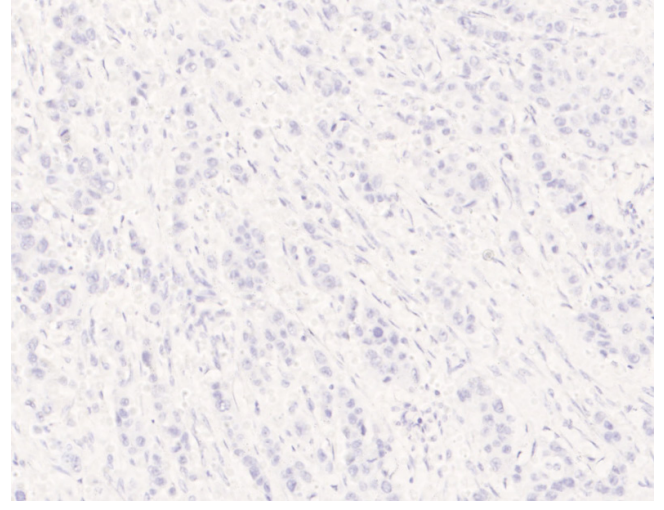

Figure 4 Breast segmental resection of case 7 . Negativity for GCDFP-15 (IHC, ×20). IHC, immunohistochemistry.

sample from a solitary mass in a 66-year-female patient (representative images of ultrasonography of case 7 seen in Figure S1). Microscopically, this patient exhibited a poorly differentiated adenocarcinoma, with scattered irregular adeno ducts in mammary parenchymal, which featured a diagnosis of primary breast cancer (seen in Figure 1). However, the patient subsequently came to consult the pathologist and provided a history of lung adenocarcinoma for 3 years and presented the original pulmonary biopsy for consultation (seen in Figure 2). Subsequently, this patient was corrected to a final diagnosis of metastatic lung adenocarcinoma to the breast by immunostaining of TTF1 (seen in Figure 3). In this scenario, the documentation of a lung cancer history would have great importance for alerting the pathologist to exclude metastatic lesions.

All of the 13 misdiagnosed cases from the enrolled 41 cases in this study were analyzed and compared in Table 2. It was found that history of prior non-mammary malignancy was of paramount importance in this context to be aware of by both clinicians and pathologists. Similarly, DeLair et al. (31) also demonstrated that the failure of recognizing the metastatic nature of the lesion by pathologist is mostly due to the absence of a prior cancer history provided by the clinician at the time of initial interpretation. The diagnostic issue becomes more prominent once a primary lung carcinoma hides behind the breast lesion. This was exemplified by case No. 7, 21,27, 29, 31 and 33. Even though a history of primary lung tumor was known, immunohistochemical staining was considered essential for differential diagnosis. Namely, a panel of markers were essential, including ER/GCDFP-15 (seen in Figure 4), or GATA-3 for breast carcinoma (36-39), and TTF-1, NapsinA 
for lung adenocarcinoma, as no single immunostaining is entirely specific to this situation (40). TTF-1, a tissuespecific transcription factor expressed in lung and thyroid epithelial cells, is expressed in approximately $72.5 \%$ (for clone SPT24) of pulmonary adenocarcinomas (41). Breast carcinoma rarely positively expresses TTF-1 (42-44), which is reported highly specific for lung adenocarcinoma, although not $100 \%$ sensitive (76-81\%) (45). Napsin-A, an aspartic proteinase, has been reported as a surrogate marker for TTF-1 in some cases (46). However, monoclonal Napsin-A strong immunoreactivity was demonstrated in $14.6 \%$ of cases of breast carcinomas with apocrine changes only (47). Thus, sole immunostaining of Napsin-A recommended for explanation of a breast metastatic lesion from the lung. In our study, three cases (3/41, 73.2\%) showed TTF1-negative staining, which were diagnosed as metastatic lesion based on clinical information, such as a known lung cancer history, widespread disease situation, and negative breast markers and hormone receptors. ER, is a signature of luminal type of breast carcinoma, with a reported highly positive rate up to $80 \%$ (48). Although lung adenocarcinomas were also reported stained with as a wide range of ER positivity depending on different staining protocols $(41,49,50)$, combined breast-specific markers (GCDFP-15, or GATA-3) would be helpful to confirm the breast origin of the specimen. None of our cases expressed GCDFP-15, or GATA-3 in keeping with the nonmammary origin. However, the triple negative entities for breast carcinoma (ER, PR negative and HER-2 negative) were reported a lower positive percentage of breast lineage markers (Mammaglobin 17-18\%, GCDFP-15 10-33\%, GATA-3 43-66\%) (41,42,51,52), which also renders a diagnostic pitfall. On the other hand, GATA-3, the most sensitive immunostaining markers for breast origin, was also reported positive staining in $8-12 \%$ of lung adenocarcinomas (53). So, a combined panel of both breastand lung-origin would be superior to a single biomarker for making a correct differential diagnosis.

In addition to morphological and IHC methods, genetic alterations for lung adenocarcinoma may also play a role in difficult differential diagnosis. In advanced lung adenocarcinoma, EGFR mutation, $A L K$ rearrangement, ROS1 fusion were routinely tested in diagnostic settings, and it is reported that these molecular subtypes are highly consistent in the primary and metastatic lesions. Thus, the detection of these molecules from the biopsy samples of suspected breast metastases and the comparison with the primary lung lesions will support the diagnosis of metastatic cancer (26). This was exemplified by cases No. 5 , 7, 39 and 40 .

In conclusion, we retrospectively reviewed a relatively large sample of metastatic adenocarcinoma from the lung to the breast, and analyzed the diagnostic dilemma. The pathogenesis of lung cancer metastasis to breast has not been reported. It is difficult to distinguish a metastatic breast mass only based on macroscopy, mammography, ultrasonography, or CT scan. Pathological examination remains gold standard for differentiation diagnosis. Also noted in our cohort, breast lesions with contralateral or no ipsilateral axillary lymphadenopathy, triple negative breast pathology, and de novo metastatic breast cancers resistant or refractory to conventional primary breast carcinoma therapeutics may add metastatic consideration to the differential diagnosis list. A documented history of lung cancer or a thorough detection of lung is essential for making a correct diagnosis. Additional breast- and lunglineage immunostaining plus genetic alternation analysis may confirm the diagnosis and guide precise treatment.

\section{Compliance with ethical standards}

All individuals listed as authors have contributed to the conception and design of the work, data acquisition, analysis, and interpretation of data. All authors had input in drafting the work and revising it critically for important intellectual content as well as approved the final version herein.

\section{Acknowledgments}

Funding: Supported by the grant 2017YFC1311005 and 2017YFC1308700 from the National Key R\&D Program of China, Beijing Municipal Science \& Technology Commission.

\section{Footnote}

Reporting Checklist: The authors have completed the STROBE reporting checklist. Available at http://dx.doi. org/10.21037/tcr-20-2250

Data Sharing Statement: Available at http://dx.doi. org/10.21037/tcr-20-2250

Peer Review File: Available at http://dx.doi.org/10.21037/tcr20-2250 
Conflicts of Interest: All authors have completed the ICMJE uniform disclosure form (available at http://dx.doi. org/10.21037/tcr-20-2250). The authors have no conflicts of interest to declare.

Ethical Statement: The authors are accountable for all aspects of the work in ensuring that questions related to the accuracy or integrity of any part of the work are appropriately investigated and resolved. The study was conducted in accordance with the Declaration of Helsinki (as revised in 2013). The study was approved by the Ethics Committee board of National Cancer Center/National Clinical Research Center for Cancer/Cancer Hospital (NCC-004409) and individual consent for this retrospective analysis was waived.

Open Access Statement: This is an Open Access article distributed in accordance with the Creative Commons Attribution-NonCommercial-NoDerivs 4.0 International License (CC BY-NC-ND 4.0), which permits the noncommercial replication and distribution of the article with the strict proviso that no changes or edits are made and the original work is properly cited (including links to both the formal publication through the relevant DOI and the license). See: https://creativecommons.org/licenses/by-nc-nd/4.0/.

\section{References}

1. Sánchez de Cos Escuín J, Abal Arca J, Melchor Íñiguez $\mathrm{R}$, et al. Tumor, node and metastasis classification of lung cancer-M1a versus M1b--analysis of $M$ descriptors and other prognostic factors. Lung Cancer 2014;84:182-9.

2. Abrams HL, Spiro R, Goldstein N. Metastases in carcinoma; analysis of 1000 autopsied cases. Cancer 1950;3:74-85.

3. Hajdu SI, Urban JA. Cancers metastatic to the breast. Cancer 1972;29:1691-6.

4. Toombs BD, Kalisher L. Metastatic disease to the breast: clinical, pathologic, and radiographic features. AJR Am J Roentgenol 1977;129:673-6.

5. McCrea ES, Johnston C, Haney PJ. Metastases to the breast. AJR Am J Roentgenol 1983;141:685-90.

6. Babu KS, Roberts F, Bryden F, et al. Metastases to breast from primary lung cancer. J Thorac Oncol 2009;4:540-2.

7. Klingen TA, Chen Y, Gundersen MD, et al. Thyroid transcription factor-1 positive primary breast cancer: a case report with review of the literature. Diagn Pathol 2010;5:37.
8. Fukumoto K, Usami N, Okasaka T, et al. Late breast metastasis from resected lung cancer diagnosed by epidermal growth factor receptor gene mutation. Lung Cancer 2011;74:352-3.

9. Ali RH, Taraboanta C, Mohammad T, et al. Metastatic non-small cell lung carcinoma a mimic of primary breast carcinoma—case series and literature review. Virchows Arch 2018;472:771-7.

10. Verger E, Conill C, Velasco M, et al. Metastasis in the male breast from a lung adenocarcinoma. Acta Oncol 1992;31:479.

11. Yeh CN, Lin CH, Chen MF. Clinical and ultrasonographic characteristics of breast metastases from extramammary malignancies. Am Surg 2004;70:287-90.

12. Rimner A, Rosenzweig KE. Palliative radiation for lung cancer metastases to the breast: two case reports. J Thorac Oncol 2007;2:1133-5.

13. Ucar N, Kurt OK, Alpar S, et al. Breast metastasis in a male patient with nonsmall cell lung carcinoma. South Med J 2007;100:850-1.

14. Klingen TA, Klaasen $\mathrm{H}$, Aas $\mathrm{H}$, et al. Secondary breast cancer: a 5-year population-based study with review of the literature. Apmis 2009;117:762-7.

15. Yoon MY, Song CS, Seo MH, et al. A case of metachronous metastasis to the breast from non-small cell lung carcinoma. Cancer Res Treat 2010;42:172-5.

16. Nasit Jitendra G, Parikh B, Shah M. Bilateral breast metastasis from an adenocarcinoma of lung: a case report. Natl J Med Res 2011;1:83-6.

17. Branica BV, Meniga IN, Puljić I, et al. Breast metastasis from lung adenocarcinoma diagnosed with fine needle aspiration cytology: a case report. Coll Antropol 2012;36:1461-5.

18. Ko K, Ro JY, Hong EK, et al. Micropapillary lung cancer with breast metastasis simulating primary breast cancer due to architectural distortion on images. Korean J Radiol 2012;13:249-53.

19. Sato K, Takeyama Y, Yoshihara M, et al. CBDCA + Pemetrexed + Bevacizumab and its maintenance chemotherapy in a case of solitary breast metastasis from a lung adenocarcinoma resistant to Gefitinib. Case Rep Oncol 2012;5:546-53.

20. Mirrielees JA, Kapur JH, Szalkucki LM, et al. Metastasis of primary lung carcinoma to the breast: a systematic review of the literature. J Surg Res 2014;188:419-31.

21. Sadikot RT, Renwick DS, Dacosta P, et al. Breast metastasis from non-small cell lung cancer. South Med J 1997;90:1063-64. 
22. Maounis N, Chorti M, Legaki S, et al. Metastasis to the breast from an adenocarcinoma of the lung with extensive micropapillary component: a case report and review of the literature. Diagn Pathol 2010;5:82.

23. Ji FF, Gao P, Wang JG, et al. Contralateral breast metastasis from pulmonary adenocarcinoma: Two cases report and literature review. J Thorac Dis 2012;4:384-389.

24. Sanguinetti A, Puma F, Lucchini R, et al. Breast metastasis from a pulmonary adenocarcinoma: case report and review of the literature. Oncol Lett 2013;5:328-32.

25. Malek D, Buccheri S, Dey CB, et al. Lung cancer metastasis to the breast mimicking inflammatory breast carcinoma on imaging. Radiol Case Rep 2019;14:1500-5.

26. Wu X, Wang H, Fang M, et al. ALK or ROS1-rearranged breast metastasis from lung adenocarcinoma: a report of 2 cases. Tumori 2019;105:NP67-71.

27. Erhamamci S, Reyhan M, Canpolat T, et al. A case of a man with isolated breast metastasis from lung adenocarcinoma incidentally detected by FDG PET/CT. Clin Nucl Med 2016;41:e146-8.

28. Georgiannos SN, Chin J, Goode AW, et al. Secondary neoplasms of the breast: a survey of the 20th century. Cancer 2001;92:2259-66.

29. Noguera J, Martínez-Miravete P, Idoate F, et al. Metastases to the breast: a review of 33 cases. Australas Radiol 2007;51:133-8.

30. Shukla R, Pooja B, Radhika S, et al. Fine-needle aspiration cytology of extramammary neoplasms metastatic to the breast. Diagn Cytopathol 2005;32:193-7.

31. DeLair DF, Corben AD, Catalano JP, et al. Non-mammary metastases to the breast and axilla: a study of 85 cases. Mod Pathol 2013;26:343-9.

32. Lee AH. The histological diagnosis of metastases to the breast from extramammary malignancies. J Clin Pathol 2007;60:1333-41.

33. Sauer T. Fine-needle aspiration cytology of extra mammary metastatic lesions in the breast: a retrospective study of 36 cases diagnosed during 18 years. Cytojournal 2010;7:10.

34. Vizcaíno I, Torregrosa A, Higueras V, et al. Metastasis to the breast from extramammary malignancies: a report of four cases and a review of literature. Eur Radiol 2001;11:1659-65.

35. Wood B, Sterrett G, Frost F, et al. Diagnosis of extramammary malignancy metastatic to the breast by fine needle biopsy. Pathology 2008;40:345-51.

36. Huo L, Gong Y, Guo M, et al. GATA-binding protein 3 enhances the utility of gross cystic disease fluid protein-15 and mammaglobin A in triple-negative breast cancer by immunohistochemistry. Histopathology 2015;67:245-54.

37. Cimino-Mathews A, Subhawong AP, Illei PB, et al. GATA3 expression in breast carcinoma: utility in triplenegative, sarcomatoid, and metastatic carcinomas. Hum Pathol 2013;44:1341-9.

38. Gown AM, Fulton RS, Kandalaft PL. Markers of metastatic carcinoma of breast origin. Histopathology 2016;68:86-95.

39. Krings G, Nystrom M, Mehdi I, et al. Diagnostic utility and sensitivities of GATA3 antibodies in triple-negative breast cancer. Hum Pathol 2014;45:2225-32.

40. Yang M, Nonaka D. A study of immunohistochemical differential expression in pulmonary and mammary carcinomas. Mod Pathol 2010;23:654-61.

41. Ordóñez NG. Value of thyroid transcription factor-1 immunostaining in tumor diagnosis: a review and update. Appl Immunohistochem Mol Morphol 2012;20:429-44.

42. Sakurai A, Sakai Y, Yatabe Y. Thyroid transcription factor-1 expression in rare cases of mammary ductal carcinoma. Histopathology 2011;59:145-8.

43. Robens J, Goldstein L, Gown AM, et al. Thyroid transcription factor-1 expression in breast carcinomas. Am J Surg Pathol 2010;34:1881-5.

44. Ni YB, Tsang JYS, Shao MM, et al. TTF-1 expression in breast carcinoma: an unusual but real phenomenon. Histopathology 2014;64:504-11.

45. Tran L, Mattsson JS, Nodin B, et al. Various antibody clones of Napsin A, thyroid transcription factor 1 , and p40 and comparisons with cytokeratin 5 and p63 in histopathologic diagnostics of non-small cell lung carcinoma. Appl Immunohistochem Mol Morphol 2016;24:648-59.

46. Ye J, Findeis-Hosey JJ, Yang Q, et al. Combination of Napsin-A and TTF-1 immunohistochemistry helps in differentiating primary lung adenocarcinoma from metastatic carcinoma in the lung. Appl Immunohistochem Mol Morphol 2011;19:313-7.

47. Vitkovski T, Chaudhary S, Sison C, et al. Aberrant expression of Napsin-A in breast carcinoma with apocrine features. Int J Surg Pathol 2016;24:377-81.

48. Lakhani SR, Ellis IO, Schnitt SJ, et al. editors. WHO classification of tumours of the breast. Lyon: IARC Press, 2012.

49. Dabbs DJ, Landreneau RJ, Liu Y, et al. Detection of estrogen receptor by immunohistochemistry in pulmonary adenocarcinoma. Ann Thorac Surg 2002;73:403-5; discussion 406. 
50. Hattori Y, Yoshida A, Yoshida M, et al. Evaluation of androgen receptor and GATA binding protein 3 as immunohistochemical markers in the diagnosis of metastatic breast carcinoma to the lung. Pathol Int 2015;65:286-92.

51. El Hag MI, Hag AM, Ha JP, et al. Comparison of GATA3, mammaglobin, GCDFP-15 expression in breast carcinoma in serous effusions: A cell-block micro-array study. Pleura Peritoneum 2017;2:143-8.

Cite this article as: Wang X, Luo Y, Liu L, Wei J, Lei H, Shi S, Yang L. Metastatic adenocarcinoma to the breast from the lung simulates primary breast carcinoma-a clinicopathologic study. Transl Cancer Res 2021;10(3):1399-1409. doi: 10.21037/tcr-20-2250
52. Kandalaft PL, Simon RA, Isacson C, et al. Comparative sensitivities and specificities of antibodies to breast markers GCDFP-15, Mammaglobin A, and different clones of antibodies to GATA-3. Appl Immunohistochem Mol Morphol 2016;24:609-14.

53. Miettinen M, McCue PA, Sarlomo-Rikala M, et al. GATA3: a multispecific but potentially useful marker in surgical pathology: a systematic analysis of 2500 epithelial and nonepithelial tumors. Am J Surg Pathol 2014;38:13-22. 
Supplementary

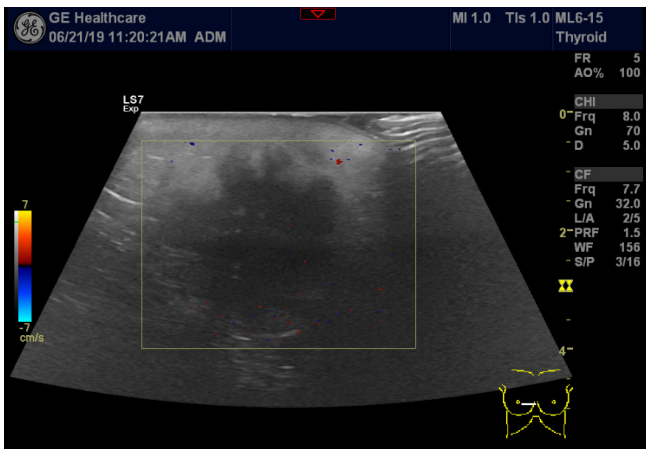

Figure S1 Representative images of ultrasonography for $\mathrm{PBC}$ of case 7. $\mathrm{PBC}$, primary invasive breast carcinoma. 